\title{
Article \\ On New Generalized Dunkel Type Integral Inequalities with Applications
}

\author{
Dong-Sheng Wang ${ }^{1}\left(\mathbb{D}\right.$, Huan-Nan $\mathrm{Shi}^{2}{ }^{\mathbb{D}}$, Chun-Ru Fu ${ }^{3}\left(\mathbb{C}\right.$ and Wei-Shih $\mathrm{Du}^{4, *} \mathbb{( 1 )}$ \\ 1 Basic Courses Department, Beijing Polytechnic, Beijing 100176, China; wangdongshen@bpi.edu.cn \\ 2 Department of Electronic Information, Teacher's College, Beijing Union University, Beijing 100011, China; \\ sfthuannan@buu.edu.cn \\ 3 Institute of Mathematics and Physics, Beijing Union University, Beijing 100101, China; bytchunru@buu.edu.cn \\ 4 Department of Mathematics, National Kaohsiung Normal University, Kaohsiung 82444, Taiwan \\ * Correspondence: wsdu@mail.nknu.edu.tw
}

check for

updates

Citation: Wang, D.-S.; Shi, H.-N.;

Fu, C.-R.; Du, W.-S. On New

Generalized Dunkel Type Integral

Inequalities with Applications.

Symmetry 2021, 13, 1576. https:/ /

doi.org/10.3390/sym13091576

Academic Editor: Paolo Emilio Ricci

Received: 6 August 2021

Accepted: 23 August 2021

Published: 27 August 2021

Publisher's Note: MDPI stays neutral with regard to jurisdictional claims in published maps and institutional affiliations.

\section{Copyright: (c) 2021 by the authors.} Licensee MDPI, Basel, Switzerland. This article is an open access article distributed under the terms and conditions of the Creative Commons Attribution (CC BY) license (https:// creativecommons.org/licenses/by/ $4.0 /)$.

\begin{abstract}
In this paper, by applying majorization theory, we study the Schur convexity of functions related to Dunkel integral inequality. We establish some new generalized Dunkel type integral inequalities and their applications to inequality theory.
\end{abstract}

Keywords: Dunkel type integral inequality; Schur-convexity; majorization theory; arithmetic meangeometric mean (AM-GM) inequality

MSC: 26A51; 26E60; 26D15; 26D05; 34K38; 39B62

\section{Introduction and Preliminaries}

Over the last half a century, rapid developments in inequality theory and its applications have contributed greatly to many branches of mathematics such as linear and nonlinear analysis, differential equations, finance, statistics, physics, fractional calculus, and so on; for more details, one can refer to [1-4] and the references therein.

The original Dunkel integral inequality can be stated as follows.

Theorem 1 (original Dunkel integral inequality; see [1-3,5,6]). Let $f(x)$ be a continuous real-valued function on $[a, b]$ which is not identically zero and satisfies $0 \leq f(x) \leq M$ for all $x \in[a, b]$. Then

$$
0<\left(\int_{a}^{b} f(x) d x\right)^{2}-\left(\int_{a}^{b} f(x) \cos x d x\right)^{2}-\left(\int_{a}^{b} f(x) \sin x d x\right)^{2} \leq \frac{1}{12} M^{2}(b-a)^{2}
$$

There are many ways to prove Dunkel integral inequality (see [1-3,5,6] and references therein). Some interesting proofs of Dunkel integral inequality are the probabilistic method (see, e.g., [1]), re-integral method (see [2,3]), and so on.

In fact, if $f(x)$ is a nonnegative continuous real-valued function on $[a, b]$ (here, $f$ is allowed to be a zero function), then from (1) one deduces the following fascinating concise inequality:

$$
\left(\int_{a}^{b} f(x) \cos x d x\right)^{2}+\left(\int_{a}^{b} f(x) \sin x d x\right)^{2} \leq\left(\int_{a}^{b} f(x) d x\right)^{2}
$$

In 1923, Professor Issai Schur first systematically studied the functions preserving the ordering of majorization. In Schur's honor, such functions are named to have "Schurconvexity". During the previous more than four decades, majorization theory and Schurconvexity have been applied widely in many areas of mathematics including integral inequality, stochastic matrices, rearrangement theory, analytic inequalities, information 
theory, quantum correlations, quantum cryptography, combinatorial optimization, and other related fields (see, e.g., [7-12]).

Let us recall some basic definitions and notation that will be needed in this paper.

Definition 1 (see $[4,8])$. Let $\Omega$ be a nonempty subset of $\mathbb{R}^{n}$.

(i) Let $x=\left(x_{1}, \ldots, x_{n}\right)$ and $y=\left(y_{1}, \ldots, y_{n}\right) \in \mathbb{R}^{n} . x$ is said to be majorized by $y$ (in symbols $x \prec y)$ if $\sum_{i=1}^{k} x_{[i]} \leq \sum_{i=1}^{k} y_{[i]}$ for $k=1,2, \ldots, n-1$ and $\sum_{i=1}^{n} x_{i}=\sum_{i=1}^{n} y_{i}$, where $x_{[1]} \geq \cdots \geq x_{[n]}$ and $y_{[1]} \geq \cdots \geq y_{[n]}$ are rearrangements of $x$ and $y$ in a descending order;

(ii) $\Omega$ is called convex if $\alpha x+\beta y \in \Omega$ for any $x, y \in \Omega$ and $\alpha, \beta \geq 0$ with $\alpha+\beta=1$;

(iii) $\Omega$ is called symmetric if $x \in \Omega$ implies $P x \in \Omega$ for every $n \times n$ permutation matrix $P$;

(iv) A function $\varphi: \Omega \rightarrow \mathbb{R}$ is called symmetric if for every permutation matrix $P, \varphi(P x)=\varphi(x)$ for all $x \in \Omega$;

(v) A function $\varphi: \Omega \rightarrow \mathbb{R}$ is said to be Schur convex on $\Omega$ if $x \prec y$ on $\Omega$ implies $\varphi(x) \leq \varphi(y)$. $\varphi$ is said to be Schur concave on $\Omega$ if and only if $-\varphi$ is Schur convex.

The paper is divided into five sections. In Sections 2 and 3, by applying majorization theory, we present some new generalized Dunkel type integral inequalities and new Dunkel ( $p$ )-type integral inequalities for $p \geq 2$. As applications of our new results, some new integral inequalities are established in Section 4. Finally, some summary and conclusions are given in Section 5.

\section{Some Generalizations of Dunkel Integral Inequality}

The following two known results are important for proving our new theorem.

Lemma 1 (see [4]). Let $a \leq b$. Let $u(t):=t a+(1-t) b$ and $v(t):=t b+(1-t) a$ for $\frac{1}{2} \leq t_{1} \leq t_{2} \leq 1$ or $0 \leq t_{1} \leq t_{2} \leq \frac{1}{2}$. Then

$$
\left(\frac{a+b}{2}, \frac{a+b}{2}\right) \prec\left(u\left(t_{2}\right), v\left(t_{2}\right)\right) \prec\left(u\left(t_{1}\right), v\left(t_{1}\right)\right) \prec(a, b) .
$$

Lemma 2 (see [4,7]). Let $\Omega \subset \mathbb{R}^{n}$ be a nonempty convex set and has a nonempty interior set $\Omega^{\circ}$. Let $\varphi: \Omega \rightarrow \mathbb{R}$ be continuous on $\Omega$ and differentiable in $\Omega^{\circ}$. Then, $\varphi$ is a Schur convex (resp. Schur concave) function, if and only if it is symmetric on $\Omega$ and

$$
\left(x_{1}-x_{2}\right)\left(\frac{\partial \varphi}{\partial x_{1}}-\frac{\partial \varphi}{\partial x_{2}}\right) \geq 0 \quad(\text { resp. } \leq 0)
$$

holds for any $x=\left(x_{1}, \cdots, x_{n}\right) \in \Omega^{\circ}$.

Remark 1. It is worth noticing that Lemma 2 is equivalent to the following: $\varphi$ is a Schur convex (resp. Schur concave) function, if and only if it is symmetric on $\Omega$ and

$$
\frac{\partial \varphi}{\partial x_{i}} \geq \frac{\partial \varphi}{\partial x_{i+1}} \quad(\text { resp. } \leq 0), i=1,2, \ldots, n-1 .
$$

for all $x \in D \cap \Omega$, where $D=\left\{x: x_{1} \geq \cdots \geq x_{n}\right\}$.

With the help of Lemmas 1 and 2, we can establish the following crucial result. 
Theorem 2. Let I be an interval of $\mathbb{R}$. Assume that $f(x)$ and $g(x)$ are two nonnegative continuous real-valued functions on $I$, and $\kappa(x)$ and $\lambda(x)$ are two continuous real-valued functions on $I$. Define $L: I \times I \rightarrow \mathbb{R}$ by

$$
\begin{aligned}
L(a, b)= & \int_{a}^{b} f(x) d x \int_{a}^{b} g(x) d x \\
& -\int_{a}^{b} f(x) \kappa(x) d x \int_{a}^{b} g(x) \kappa(x) d x-\int_{a}^{b} f(x) \lambda(x) d x \int_{a}^{b} g(x) \lambda(x) d x
\end{aligned}
$$

for any $(a, b) \in I \times I$. Then the following holds:

(i) If $\kappa(x) \cdot \kappa(b)+\lambda(x) \cdot \lambda(b) \leq 1$ and $\kappa(x) \cdot \kappa(a)+\lambda(x) \cdot \lambda(a) \leq 1$ for $x, a, b \in I$, then $L$ is Schur convex on $I \times I$;

(ii) If $\kappa(x) \cdot \kappa(b)+\lambda(x) \cdot \lambda(b) \geq 1$ and $\kappa(x) \cdot \kappa(a)+\lambda(x) \cdot \lambda(a) \geq 1$ for $x, a, b \in I$, then L is Schur concave on $I \times I$.

Proof. Obviously, $L(a, b)$ is a symmetric operator for $a, b \in I$. So, without loss of generality, we may assume that $b \geq a$. Since

$$
\begin{aligned}
\frac{\partial L}{\partial b}= & f(b) \int_{a}^{b} g(x) d x+g(b) \int_{a}^{b} f(x) d x \\
& -f(b) \kappa(b) \int_{a}^{b} g(x) \kappa(x) d x-g(b) \kappa(b) \int_{a}^{b} f(x) \kappa(x) d x \\
& -f(b) \lambda(b) \int_{a}^{b} g(x) \lambda(x) d x-g(b) \lambda(b) \int_{a}^{b} f(x) \lambda(x) d x
\end{aligned}
$$

and

$$
\begin{aligned}
\frac{\partial L}{\partial a}= & -f(a) \int_{a}^{b} g(x) d x-g(a) \int_{a}^{b} f(x) d x \\
& +f(a) \kappa(a) \int_{a}^{b} g(x) \kappa(x) d x+g(a) \kappa(a) \int_{a}^{b} f(x) \kappa(x) d x \\
& +f(a) \lambda(a) \int_{a}^{b} g(x) \lambda(x) d x+g(a) \lambda(a) \int_{a}^{b} f(x) \lambda(x) d x,
\end{aligned}
$$

we have

$$
\begin{aligned}
\Delta:= & (b-a)\left(\frac{\partial L}{\partial b}-\frac{\partial L}{\partial a}\right) \\
= & (f(a)+f(b)) \int_{a}^{b} g(x) d x+(g(a)+g(b)) \int_{a}^{b} f(x) d x \\
& -(f(b) \kappa(b)+f(a) \kappa(a)) \int_{a}^{b} g(x) \kappa(x) d x-(f(b) \lambda(b)+f(a) \lambda(a)) \int_{a}^{b} g(x) \lambda(x) d x \\
& -(g(b) \kappa(b)+g(a) \kappa(a)) \int_{a}^{b} f(x) \kappa(x) d x-(g(b) \lambda(b)+g(a) \lambda(a)) \int_{a}^{b} f(x) \lambda(x) d x \\
= & f(b) \int_{a}^{b} g(x)(1-\kappa(b) \kappa(x)-\lambda(b) \lambda(x)) d x+f(a) \int_{a}^{b} g(x)(1-\kappa(a) \kappa(x) \\
& -\lambda(a) \lambda(x)) d x+g(b) \int_{a}^{b} f(x)(1-\kappa(b) \kappa(x)-\lambda(b) \lambda(x)) d x \\
& +g(a) \int_{a}^{b} f(x)(1-\kappa(a) \kappa(x)-\lambda(a) \lambda(x)) d x .
\end{aligned}
$$

(i) When $\kappa(x) \cdot \kappa(b)+\lambda(x) \cdot \lambda(b) \leq 1$ and $\kappa(x) \cdot \kappa(a)+\lambda(x) \cdot \lambda(a) \leq 1$, we have $\Delta \geq 0$. By Lemma 2, $L$ is Schur convex on $I \times I$. 
(ii) When $\kappa(x) \cdot \kappa(b)+\lambda(x) \cdot \lambda(b) \geq 1$ and $\kappa(x) \cdot \kappa(a)+\lambda(x) \cdot \lambda(a) \geq 1$, we have $\Delta \leq 0$. By Lemma $2, L$ is Schur concave on $I \times I$.

The proof is completed.

We now present the following generalized Dunkel type integral inequality which is one of the main results of this paper.

Theorem 3. Let I be an interval of $\mathbb{R}$. Assume that $f(x)$ and $g(x)$ are two nonnegative continuous real-valued functions on $I$, and $\kappa(x)$ and $\lambda(x)$ are two continuous real-valued functions on $I$. Let $u(t):=t a+(1-t) b$ and $v(t):=t b+(1-t) a$, for $\frac{1}{2} \leq t \leq 1$. Then the following holds:

(i) If $\kappa(x) \cdot \kappa(b)+\lambda(x) \cdot \lambda(b) \leq 1$ and $\kappa(x) \cdot \kappa(a)+\lambda(x) \cdot \lambda(a) \leq 1$ for $x, a, b \in I$, then

$$
\begin{aligned}
& \int_{a}^{b} f(x) \kappa(x) d x \int_{a}^{b} g(x) \kappa(x) d x+\int_{a}^{b} f(x) \lambda(x) d x \int_{a}^{b} g(x) \lambda(x) d x \\
\leq & \int_{a}^{b} f(x) d x \int_{a}^{b} g(x) d x-\int_{u(t)}^{v(t)} f(x) d x \int_{u(t)}^{v(t)} g(x) d x \\
& +\int_{u(t)}^{v(t)} f(x) \kappa(x) d x \int_{u(t)}^{v(t)} g(x) \kappa(x) d x+\int_{u(t)}^{v(t)} f(x) \lambda(x) d x \int_{u(t)}^{v(t)} g(x) \lambda(x) d x \\
\leq & \int_{a}^{b} f(x) d x \int_{a}^{b} g(x) d x ;
\end{aligned}
$$

(ii) If $\kappa(x) \cdot \kappa(b)+\lambda(x) \cdot \lambda(b) \geq 1$ and $\kappa(x) \cdot \kappa(a)+\lambda(x) \cdot \lambda(a) \geq 1$ for $x, a, b \in I$, then

$$
\begin{aligned}
& \int_{a}^{b} f(x) \kappa(x) d x \int_{a}^{b} g(x) \kappa(x) d x+\int_{a}^{b} f(x) \lambda(x) d x \int_{a}^{b} g(x) \lambda(x) d x \\
\geq & \int_{a}^{b} f(x) d x \int_{a}^{b} g(x) d x-\int_{u(t)}^{v(t)} f(x) d x \int_{u(t)}^{v(t)} g(x) d x \\
& +\int_{u(t)}^{v(t)} f(x) \kappa(x) d x \int_{u(t)}^{v(t)} g(x) \kappa(x) d x+\int_{u(t)}^{v(t)} f(x) \lambda(x) d x \int_{u(t)}^{v(t)} g(x) \lambda(x) d x \\
\geq & \int_{a}^{b} f(x) d x \int_{a}^{b} g(x) d x .
\end{aligned}
$$

Proof. We only show case (i) and a similar argument could be made for the case (ii). Define $L: I \times I \rightarrow \mathbb{R}$ by

$$
\begin{aligned}
L(a, b)= & \int_{a}^{b} f(x) d x \int_{a}^{b} g(x) d x \\
& -\int_{a}^{b} f(x) \kappa(x) d x \int_{a}^{b} g(x) \kappa(x) d x-\int_{a}^{b} f(x) \lambda(x) d x \int_{a}^{b} g(x) \lambda(x) d x
\end{aligned}
$$

for any $(a, b) \in I \times I$. If $\kappa(x) \cdot \kappa(b)+\lambda(x) \cdot \lambda(b) \leq 1$ and $\kappa(x) \cdot \kappa(a)+\lambda(x) \cdot \lambda(a) \leq 1$ for $x, a, b \in I$, by applying Theorem 2 (i), we show that $L$ is Schur convex on $I \times I$. On the other hand, by using Lemma 1 , we get

$$
L(a, b) \geq L(u(t), v(t)) \geq L\left(\frac{a+b}{2}, \frac{a+b}{2}\right)=0 .
$$


Hence, we obtain

$$
\begin{aligned}
& \int_{a}^{b} f(x) d x \int_{a}^{b} g(x) d x-\int_{a}^{b} f(x) \kappa(x) d x \int_{a}^{b} g(x) \kappa(x) d x \\
& -\int_{a}^{b} f(x) \lambda(x) d x \int_{a}^{b} g(x) \lambda(x) d x \\
\geq & \int_{u(t)}^{v(t)} f(x) d x \int_{u(t)}^{v(t)} g(x) d x-\int_{u(t)}^{v(t)} f(x) \kappa(x) d x \int_{u(t)}^{v(t)} g(x) \kappa(x) d x \\
& -\int_{u(t)}^{v(t)} f(x) \lambda(x) d x \int_{u(t)}^{v(t)} g(x) \lambda(x) d x \\
\geq & 0,
\end{aligned}
$$

which implies

$$
\begin{aligned}
& \int_{a}^{b} f(x) \kappa(x) d x \int_{a}^{b} g(x) \kappa(x) d x+\int_{a}^{b} f(x) \lambda(x) d x \int_{a}^{b} g(x) \lambda(x) d x \\
\leq & \int_{a}^{b} f(x) d x \int_{a}^{b} g(x) d x-\int_{u(t)}^{v(t)} f(x) d x \int_{u(t)}^{v(t)} g(x) d x \\
& +\int_{u(t)}^{v(t)} f(x) \kappa(x) d x \int_{u(t)}^{v(t)} g(x) \kappa(x) d x+\int_{u(t)}^{v(t)} f(x) \lambda(x) d x \int_{u(t)}^{v(t)} g(x) \lambda(x) d x \\
\leq & \int_{a}^{b} f(x) d x \int_{a}^{b} g(x) d x .
\end{aligned}
$$

The proof is completed.

As a direct consequence of Theorem 3, we can obtain the following generalized Dunkel integral inequality.

Theorem 4 (Generalized Dunkel integral inequality). Let $f(x)$ and $g(x)$ be two nonnegative continuous real-valued functions on $[a, b]$ and $m$ be any real number. Then

$$
\begin{aligned}
& \left(\int_{a}^{b} f(x) \cos m x d x\right)\left(\int_{a}^{b} g(x) \cos m x d x\right)+\left(\int_{a}^{b} f(x) \sin m x d x\right)\left(\int_{a}^{b} g(x) \sin m x d x\right) \\
& \leq\left(\int_{a}^{b} f(x) d x\right)\left(\int_{a}^{b} g(x) d x\right) .
\end{aligned}
$$

Proof. In theorem 3, we take $I=[a, b], \kappa(x)=\cos m x$, and $\lambda(x)=\sin m x$ for $x \in I$. Thus, $\kappa(x)$ and $\lambda(x)$ are two continuous real-valued functions on $I$. Clearly, we have

$$
\begin{aligned}
\kappa(x) \cdot \kappa(b)+\lambda(x) \cdot \lambda(b) & =\cos m x \cdot \cos m b+\sin m x \cdot \sin m b \\
& =\cos m(b-x) \leq 1
\end{aligned}
$$

and

$$
\begin{aligned}
\kappa(x) \cdot \kappa(a)+\lambda(x) \cdot \lambda(a) & =\cos m x \cdot \cos m a+\sin m x \cdot \sin m a \\
& =\cos m(a-x) \leq 1 .
\end{aligned}
$$

Thus, all the assumptions of Theorem 3 (i) are satisfied. Therefore the desired conclusion follows immediately from Theorem 3.

The following generalized Dunkel integral inequality is an immediate consequence of Theorem 4. 
Corollary 1 (Generalized Dunkel integral inequality). Let $f(x)$ be a continuous nonnegative real-valued function on $[a, b]$ and $m$ be any real number. Then

$$
\left(\int_{a}^{b} f(x) \cos m x d x\right)^{2}+\left(\int_{a}^{b} f(x) \sin m x d x\right)^{2} \leq\left(\int_{a}^{b} f(x) d x\right)^{2}
$$

Remark 2. It is worth noticing that inequality (3) in Theorem 4 and inequality (4) in Corollary 1 are real generalizations of inequality (2).

\section{A New Dunkel ( $p$ )-Type Integral Inequality for $p \geq 2$}

In this section, we will present a new Dunkel ( $p$ )-type integral inequality for $p \geq 2$. In order to prove our results, we need the following important auxiliary lemma.

Lemma 3. Let $k \in \mathbb{N} \cup\{0\}$. Denote $I_{k}:=\left[2 k \pi, 2 k \pi+\frac{\pi}{2}\right]$. Assume that $f(x)$ is a nonnegative continuous real-valued function on $I_{k}$. Define $M: I_{k} \times I_{k} \rightarrow \mathbb{R}$ by

$$
\begin{aligned}
M(a, b)= & {\left[(b-a) \int_{a}^{b} f(x) \sin x d x\right]^{p} } \\
& +\left[(b-a) \int_{a}^{b} f(x) \cos x d x\right]^{p}-\left[(b-a) \int_{a}^{b} f(x) d x\right]^{p}
\end{aligned}
$$

for $(a, b) \in I_{k} \times I_{k}$. If $p \geq 2$, then $M$ is Schur concave on $I_{k} \times I_{k}$.

Proof. It is obvious that $M(a, b)$ is symmetric for $a, b$. Hence, without loss of generality, we may assume that $b \geq a$. By Corollary 1 , we have

$$
\begin{aligned}
\frac{\partial M}{\partial b} & =p\left[(b-a) \int_{a}^{b} f(x) \sin x d x\right]^{p-1}\left[\int_{a}^{b} f(x) \sin x d x+(b-a) f(b) \sin b\right] \\
& +p\left[(b-a) \int_{a}^{b} f(x) \cos x d x\right]^{p-1}\left[\int_{a}^{b} f(x) \cos x d x+(b-a) f(b) \cos b\right] \\
& -p\left[(b-a) \int_{a}^{b} f(x) d x\right]^{p-1}\left[\int_{a}^{b} f(x) d x+(b-a) f(b)\right] \\
& =p(b-a)^{p-1}\left[\int_{a}^{b} f(x) \sin x d x\right]^{p-2} \\
& \times\left[\left(\int_{a}^{b} f(x) \sin x d x\right)^{2}+(b-a) f(b) \sin b \int_{a}^{b} f(x) \sin x d x\right] \\
& +p(b-a)^{p-1}\left[\int_{a}^{b} f(x) \cos x d x\right]^{p-2} \\
& \times\left[\left(\int_{a}^{b} f(x) \cos x d x\right)^{2}+(b-a) f(b) \cos b \int_{a}^{b} f(x) \cos x d x\right] \\
& -p(b-a)^{p-1}\left[\int_{a}^{b} f(x) d x\right]^{p-2}\left[\left(\int_{a}^{b} f(x) d x\right)^{2}+(b-a) f(b) \int_{a}^{b} f(x) d x\right]
\end{aligned}
$$




$$
\begin{aligned}
& \leq p(b-a)^{p-1}\left(\int_{a}^{b} f(x) d x\right)^{p-2} \\
& \times\left[\left(\int_{a}^{b} f(x) \sin x d x\right)^{2}+\left(\int_{a}^{b} f(x) \cos x d x\right)^{2}-\left(\int_{a}^{b} f(x) d x\right)^{2}\right. \\
& \left.+(b-a) f(b) \int_{a}^{b} f(x)(\sin b \cdot \sin x+\cos b \cdot \cos x-1) d x\right] \\
& =p(b-a)^{p-1}\left(\int_{a}^{b} f(x) d x\right)^{p-2} \\
& \times\left[\left(\int_{a}^{b} f(x) \sin x d x\right)^{2}+\left(\int_{a}^{b} f(x) \cos x d x\right)^{2}-\left(\int_{a}^{b} f(x) d x\right)^{2}\right. \\
& \left.\left.+(b-a) f(b) \int_{a}^{b} f(x)(\cos (b-x))-1\right) d x\right] \\
& \leq 0
\end{aligned}
$$

and

$$
\begin{aligned}
& \frac{\partial M}{\partial a}=p\left[(b-a) \int_{a}^{b} f(x) \sin x d x\right]^{p-1}\left[-\int_{a}^{b} f(x) \sin x d x-(b-a) f(a) \sin a\right] \\
& +p\left[(b-a) \int_{a}^{b} f(x) \cos x d x\right]^{p-1}\left[-\int_{a}^{b} f(x) \cos x d x-(b-a) f(a) \cos a\right] \\
& -p\left[(b-a) \int_{a}^{b} f(x) d x\right]^{p-1}\left[-\int_{a}^{b} f(x) d x-(b-a) f(a)\right] \\
& =-p(b-a)^{p-1}\left[\int_{a}^{b} f(x) \sin x d x\right]^{p-2} \\
& \times\left[\left(\int_{a}^{b} f(x) \sin x d x\right)^{2}+(b-a) f(a) \sin a \int_{a}^{b} f(x) \sin x d x\right] \\
& -p(b-a)^{p-1}\left[\int_{a}^{b} f(x) \cos x d x\right]^{p-2} \\
& \times\left[\left(\int_{a}^{b} f(x) \cos x d x\right)^{2}+(b-a) f(a) \cos a \int_{a}^{b} f(x) \cos x d x\right] \\
& +p(b-a)^{p-1}\left[\int_{a}^{b} f(x) d x\right]^{p-2}\left[\left(\int_{a}^{b} f(x) d x\right)^{2}+(b-a) f(a) \int_{a}^{b} f(x) d x\right] \\
& \geq-p(b-a)^{p-1}\left(\int_{a}^{b} f(x) d x\right)^{p-2} \\
& \times\left[\left(\int_{a}^{b} f(x) \sin x d x\right)^{2}+\left(\int_{a}^{b} f(x) \cos x d x\right)^{2}-\left(\int_{a}^{b} f(x) d x\right)^{2}\right. \\
& \left.+(b-a) f(a) \int_{a}^{b} f(x)(\sin a \cdot \sin x+\cos a \cdot \cos x-1) d x\right]
\end{aligned}
$$




$$
\begin{aligned}
& =-p(b-a)^{p-1}\left(\int_{a}^{b} f(x) d x\right)^{p-2} \\
& \times\left[\left(\int_{a}^{b} f(x) \sin x d x\right)^{2}+\left(\int_{a}^{b} f(x) \cos x d x\right)^{2}-\left(\int_{a}^{b} f(x) d x\right)^{2}\right. \\
& \left.\left.+(b-a) f(a) \int_{a}^{b} f(x)(\cos (a-x))-1\right) d x\right] \\
& \geq 0
\end{aligned}
$$

which deduce

$$
\Delta^{\prime}:=(b-a)\left(\frac{\partial M}{\partial b}-\frac{\partial M}{\partial a}\right) \leq 0 .
$$

By Lemma 2, $M$ is Schur concave on $I_{k} \times I_{k}$. The proof is completed.

The following result is a new Dunkel ( $p$ )-type integral inequality for $p \geq 2$.

Theorem 5. Let $k \in \mathbb{N} \cup\{0\}$. Denote $I_{k}:=\left[2 k \pi, 2 k \pi+\frac{\pi}{2}\right]$. Assume that $f(x)$ is a nonnegative continuous real-valued function on $I_{k}$. If $p \geq 2$ and $[a, b] \subseteq I_{k}$, then

$$
\left(\int_{a}^{b} f(x) \cos x d x\right)^{p}+\left(\int_{a}^{b} f(x) \sin x d x\right)^{p} \leq\left(\int_{a}^{b} f(x) d x\right)^{p} .
$$

Proof. Define $M: I_{k} \times I_{k} \rightarrow \mathbb{R}$ by

$$
\begin{aligned}
M(a, b)= & {\left[(b-a) \int_{a}^{b} f(x) \sin x d x\right]^{p} } \\
& +\left[(b-a) \int_{a}^{b} f(x) \cos x d x\right]^{p}-\left[(b-a) \int_{a}^{b} f(x) d x\right]^{p}
\end{aligned}
$$

for $(a, b) \in I_{k} \times I_{k}$. By Lemmas 1 and 3, we obtain

$$
M(a, b) \leq M\left(\frac{a+b}{2}, \frac{a+b}{2}\right)=0,
$$

which means that

$$
\left(\int_{a}^{b} f(x) \cos x d x\right)^{p}+\left(\int_{a}^{b} f(x) \sin x d x\right)^{p} \leq\left(\int_{a}^{b} f(x) d x\right)^{p} .
$$

The following result is immediate from Theorem 5 .

Corollary 2. Let $n \in \mathbb{N}$. Let $k_{1}, k_{2}, \cdots, k_{n} \in \mathbb{N} \cup\{0\}$. Assume that $f_{i}(x)$ is a nonnegative continuous real-valued function on $\left[2 k_{i} \pi, 2 k_{i} \pi+\frac{\pi}{2}\right]$ and $\left[a_{i}, b_{i}\right] \subseteq\left[2 k_{i} \pi, 2 k_{i} \pi+\frac{\pi}{2}\right]$ for any $1 \leq i \leq n$. If $p \geq 2$, then

$$
\sum_{i=1}^{n}\left(\int_{a_{i}}^{b_{i}} f_{i}(x) \cos x d x\right)^{p}+\sum_{i=1}^{n}\left(\int_{a_{i}}^{b_{i}} f_{i}(x) \sin x d x\right)^{p} \leq \sum_{i=1}^{n}\left(\int_{a_{i}}^{b_{i}} f_{i}(x) d x\right)^{p} .
$$

\section{Some New Integral Inequalities} results.

In this section, we will provide some new integral inequalities by applying our main 
Lemma 4 (Bessel inequality; see [1]). Let $f(x)$ be a continuous or a piecewise continuous nonnegative function on $[0,2 \pi]$. The Fourier series of $f(x)$ is

$$
\frac{a_{0}}{2}+\sum_{m=1}^{\infty}\left(a_{m} \cos m x+b_{m} \sin m x\right)
$$

where $a_{0}=\frac{1}{\pi} \int_{0}^{2 \pi} f(x) d x, a_{m}=\frac{1}{\pi} \int_{0}^{2 \pi} f(x) \cos m x d x$, and $b_{m}=\frac{1}{\pi} \int_{0}^{2 \pi} f(x) \sin m x d x$, for $m \in \mathbb{N}$. Then

$$
\frac{a_{0}^{2}}{2}+\sum_{m=1}^{n}\left(a_{m}^{2}+b_{m}^{2}\right) \leq \frac{1}{\pi} \int_{0}^{2 \pi} f^{2}(x) d x .
$$

Lemma 5 (see [1]). Let $f(x)$ be a nonnegative integrable concave function on $[a, b]$. If $p \geq 1$, then

$$
\int_{a}^{b} f^{p}(x) d x \leq \frac{2^{p}}{(b-a)^{p-1}(p+1)}\left(\int_{a}^{b} f(x) d x\right)^{p} .
$$

Theorem 6. Let $f(x)$ be a nonnegative continuous concave function on $[0,2 \pi]$. Then

$$
\left(\int_{0}^{2 \pi} f(x) \sin x d x\right)^{2}+\left(\int_{0}^{2 \pi} f(x) \cos x d x\right)^{2} \leq \frac{4}{9 \pi^{2}}\left(\int_{0}^{2 \pi} f(x) d x\right)^{2} .
$$

Proof. Using the notations in Lemma 4 and applying Theorem 4, we get

$$
\sum_{m=1}^{n}\left(a_{m}^{2}+b_{m}^{2}\right) \leq n a_{0}^{2}
$$

By combining (5) with Bessel inequality (see Lemma 4), we obtain

$$
\sum_{m=1}^{n}\left(a_{m}^{2}+b_{m}^{2}\right) \leq 2 n\left(\frac{1}{\pi} \int_{0}^{2 \pi} f^{2}(x) d x-\sum_{m=1}^{n}\left(a_{m}^{2}+b_{m}^{2}\right)\right)
$$

which implies

$$
\sum_{m=1}^{n}\left(a_{m}^{2}+b_{m}^{2}\right) \leq \frac{2 n}{(2 n+1) \pi} \int_{0}^{2 \pi} f^{2}(x) d x .
$$

Let $n=1$. By applying Lemma 5 , we obtain

$$
\begin{aligned}
\left(\int_{0}^{2 \pi} f(x) \sin x d x\right)^{2}+\left(\int_{0}^{2 \pi} f(x) \cos x d x\right)^{2} & \leq \frac{2}{3 \pi} \int_{0}^{2 \pi} f^{2}(x) d x \\
& \leq \frac{4}{9 \pi^{2}}\left(\int_{0}^{2 \pi} f(x) d x\right)^{2} .
\end{aligned}
$$

The proof is completed.

Theorem 7. Let $f(x)$ be a nonnegative continuous function on $[a, b]$. If $0<a \leq x \leq b<\frac{\pi}{2}$, then

$$
\begin{aligned}
& \left(\int_{a}^{b} f(x) \tan x d x\right)^{2}+\left(\int_{a}^{b} f(x) \cot x d x\right)^{2} \\
& \geq 2\left(\int_{a}^{b} f(x) d x\right)^{2}+\left(\int_{u(t)}^{v(t)} f(x) \tan x d x\right)^{2}+\left(\int_{u(t)}^{v(t)} f(x) \cot x d x\right)^{2}-2\left(\int_{u(t)}^{v(t)} f(x) d x\right)^{2} \\
& \geq 2\left(\int_{a}^{b} f(x) d x\right)^{2},
\end{aligned}
$$


where $u(t)=t a+(1-t) b$ and $v(t)=t b+(1-t) a$, for $\frac{1}{2} \leq t \leq 1$.

Proof. Let $\kappa(x)=\frac{\sqrt{2}}{2} \tan x$ and $\lambda(x)=\frac{\sqrt{2}}{2} \cot x$ for $x \in[a, b]$. By the arithmetic meangeometric mean (AM-GM) inequality, we have

$$
\begin{aligned}
\kappa(x) \cdot \kappa(b)+\lambda(x) \cdot \lambda(b) & =\frac{1}{2} \tan x \cdot \tan b+\frac{1}{2} \cot x \cdot \cot b \\
& \geq(\tan x \cdot \tan b \cdot \cot x \cdot \cot b)^{\frac{1}{2}}=1 .
\end{aligned}
$$

In the same way, we also have $\kappa(x) \cdot \kappa(a)+\lambda(x) \cdot \lambda(a) \geq 1$. By Theorem 2 (ii), we obtain

$$
\begin{aligned}
& \left(\int_{a}^{b} f(x) \frac{\sqrt{2} \tan x}{2} d x\right)^{2}+\left(\int_{a}^{b} f(x) \frac{\sqrt{2} \cot x}{2} d x\right)^{2} \\
\geq & \left(\int_{a}^{b} f(x) d x\right)^{2}+\left(\int_{u(t)}^{v(t)} f(x) \frac{\sqrt{2} \tan x}{2} d x\right)^{2} \\
& +\left(\int_{u(t)}^{v(t)} f(x) \frac{\sqrt{2} \cot x}{2} d x\right)^{2}-\left(\int_{u(t)}^{v(t)} f(x) d x\right)^{2} \\
\geq & \left(\int_{a}^{b} f(x) d x\right)^{2},
\end{aligned}
$$

which deduces

$$
\begin{aligned}
& \left(\int_{a}^{b} f(x) \tan x d x\right)^{2}+\left(\int_{a}^{b} f(x) \cot x d x\right)^{2} \\
\geq & 2\left(\int_{a}^{b} f(x) d x\right)^{2}+\left(\int_{u(t)}^{v(t)} f(x) \tan x d x\right)^{2} \\
& +\left(\int_{u(t)}^{v(t)} f(x) \cot x d x\right)^{2}-2\left(\int_{u(t)}^{v(t)} f(x) d x\right)^{2} \\
\geq & 2\left(\int_{a}^{b} f(x) d x\right)^{2} .
\end{aligned}
$$

The proof is completed.

Theorem 8. Let $0 \leq a<b \leq 1$ and $f(x)$ be a nonnegative continuous function on $[a, b]$. If $\beta \geq \frac{1}{2}$, then

$$
\left(\int_{a}^{b} x^{\beta} f(x) d x\right)^{2}+\left(\int_{a}^{b}(1-x)^{\beta} f(x) d x\right)^{2} \leq\left(\int_{a}^{b} f(x) d x\right)^{2}
$$

Proof. Let $\kappa(x)=x^{\beta}$ and $\lambda(x)=(1-x)^{\beta}$ for $x \in[a, b]$. For any $x \in[a, b]$, since $\beta \geq \frac{1}{2}$, we have

$$
\begin{aligned}
\kappa(x) \cdot \kappa(b)+\lambda(x) \cdot \lambda(b) & =x^{\beta} \cdot b^{\beta}+(1-x)^{\beta} \cdot(1-b)^{\beta} \\
& \leq x^{\frac{1}{2}} \cdot b^{\frac{1}{2}}+(1-x)^{\frac{1}{2}} \cdot(1-b)^{\frac{1}{2}} \\
& \leq \frac{x+b+1-x+1-b}{2} \\
& =1 .
\end{aligned}
$$

In the same way, we can also show that $\kappa(x) \cdot \kappa(a)+\lambda(x) \cdot \lambda(a) \leq 1$ for $x \in[a, b]$. Therefore, the desired inequality (6) follows immediately from Theorem 2 (i). 
Theorem 9. Let $p \geq 1$ and $0 \leq a<b \leq 1$. Assume that $f(x)$ is a nonnegative continuous function on $[a, b]$. If $f(x)$ is decreasing and $x f(x)$ is increasing, or $f(x)$ is increasing and $(1-x) f(x)$ is decreasing, then

$$
2 m^{p}\left(\int_{a}^{b} f(x) d x\right)^{2} \leq\left(\int_{a}^{b} x^{p} f(x) d x\right)^{2}+\left(\int_{a}^{b}(1-x)^{p} f(x) d x\right)^{2} \leq\left(\int_{a}^{b} f(x) d x\right)^{2},
$$

where $m:=\min \{a(1-a), b(1-b)\}$.

Proof. From Theorem 8, we know that the right side of the desired inequality (7) holds. Next, we verify that the left side of desired inequality (7) also holds. By the AM-GM inequality, we have

$$
\left(\int_{a}^{b} x^{p} f(x) d x\right)^{2}+\left(\int_{a}^{b}(1-x)^{p} f(x) d x\right)^{2} \geq 2 \int_{a}^{b} x^{p} f(x) d x \int_{a}^{b}(1-x)^{p} f(x) d x .
$$

Let $\varphi(x)=x^{p} f(x)$ and $\mu(x)=(1-x)^{p} f(x)$ for $x \in[a, b]$. Thus, we get

$$
\varphi^{\prime}(x)=x^{p-1}\left(p f(x)+x f^{\prime}(x)\right)
$$

and

$$
\mu^{\prime}(x)=(1-x)^{p-1}\left[-p f(x)+(1-x) f^{\prime}(x)\right] .
$$

Since $p \geq 1$, if $f(x)$ is decreasing and $x f(x)$ is increasing, we obtain $\varphi^{\prime}(x) \geq 0$ and $\mu^{\prime}(x) \leq 0$. Similarly, if $f(x)$ is increasing and $(1-x) f(x)$ is decreasing, we also have $\varphi^{\prime}(x) \geq 0$ and $\mu^{\prime}(x) \leq 0$. By the Chebyshev inequality, we have

$$
\begin{aligned}
\left(\int_{a}^{b} x^{p} f(x) d x\right)^{2}+\left(\int_{a}^{b}(1-x)^{p} f(x) d x\right)^{2} & \geq 2 \int_{a}^{b} x^{p} f(x) d x \int_{a}^{b}(1-x)^{p} f(x) d x \\
& \geq 2(b-a) \int_{a}^{b}[x(1-x)]^{p} f(x) d x .
\end{aligned}
$$

Since $h(x)=x(1-x)$ is concave, $h$ attains its minimum value $h(a)$ or $h(b)$. Due to $m=\min \{a(1-a), b(1-b)\}$, we obtain

$$
\begin{aligned}
& \left(\int_{a}^{b} x^{p} f(x) d x\right)^{2}+\left(\int_{a}^{b}(1-x)^{p} f(x) d x\right)^{2} \\
\geq & 2(b-a) \int_{a}^{b}[x(1-x)]^{p} f(x) d x \\
\geq & 2(b-a) m^{p} \int_{a}^{b} f^{2}(x) d x \\
\geq & 2 m^{p}\left(\int_{a}^{b} f(x) d x\right)^{2} .
\end{aligned}
$$

The proof is completed.

\section{Conclusions}

In this paper, we establish the following two important main results for the generalized Dunkel type integral inequality: 
- (Generalized Dunkel integral inequality; see Theorem 4.)

Let $f(x)$ and $g(x)$ be two nonnegative continuous real-valued functions on $[a, b]$ and $m$ be any real number. Then

$$
\begin{aligned}
& \left(\int_{a}^{b} f(x) \cos m x d x\right)\left(\int_{a}^{b} g(x) \cos m x d x\right)+\left(\int_{a}^{b} f(x) \sin m x d x\right)\left(\int_{a}^{b} g(x) \sin m x d x\right) \\
& \leq\left(\int_{a}^{b} f(x) d x\right)\left(\int_{a}^{b} g(x) d x\right) .
\end{aligned}
$$

- $\quad($ Dunkel ( $p$ )-type integral inequality for $p \geq 2$; see Theorem 5.)

Let $k \in \mathbb{N} \cup\{0\}$. Denote $I_{k}:=\left[2 k \pi, 2 k \pi+\frac{\pi}{2}\right]$. Assume that $f(x)$ is a nonnegative continuous real-valued function on $I_{k}$. If $p \geq 2$ and $[a, b] \subseteq I_{k}$, then

$$
\left(\int_{a}^{b} f(x) \cos x d x\right)^{p}+\left(\int_{a}^{b} f(x) \sin x d x\right)^{p} \leq\left(\int_{a}^{b} f(x) d x\right)^{p}
$$

As applications of our new results, some new integral inequalities are presented in Section 4.

Author Contributions: Writing original draft, D.-S.W., H.-N.S., C.-R.F. and W.-S.D. All authors have read and agreed to the published version of the manuscript.

Funding: The fourth author is partially supported by Grant No. MOST 110-2115-M-017-001 of the Ministry of Science and Technology of the Republic of China.

Institutional Review Board Statement: Not applicable.

Informed Consent Statement: Not applicable.

Data Availability Statement: Not applicable.

Acknowledgments: The authors wish to express their hearty thanks to the anonymous referees for their valuable suggestions and comments.

Conflicts of Interest: The authors declare no conflict of interest.

\section{References}

1. Kuang, J.C. Applied Inequalities, 4th ed.; Shandong Press of Science and Technology: Jinan, China, 2010. (In Chinese)

2. Wang, W.L. Approaches to Prove Inequalities; Press of Harbin Industrial University: Harbin, China, 2011. (In Chinese)

3. Mitrinović, D.S.; Vasić, P.M. Analytic Inequalities; Springer: Berlin/Heidelberg, Germany, 1970.

4. Shi, H.-N. Schur Convex Functions and Inequalities; Press of Harbin Industrial University: Harbin, China, 2017. (In Chinese)

5. Dunkel, O.; Bennett, A.A. Problem 3104. Am. Math. Mon. 1925, 32, 319-321. [CrossRef]

6. Wang, W.L.; Zhang, Q. Dunkel type inequalities involving hyperbolic functions. Proc. Jangjeon Math. Soc. 2018, 21, 205-219.

7. Wang, B.Y. Foundations of Majorization Inequalities; Press of Beijing Normal Univ: Beijing, China, 1990. (In Chinese)

8. Marshall, A.M.; Olkin, I. Inequalities: Theory of Majorization and Its Application; Academies Press: New York, NY, USA, 1979.

9. Marshall, A.W.; Olkin, I.; Arnold, B.C. Inequalities: Theory of Majorization and Its Application, 2nd ed.; Springer: New York, NY, USA, 2011.

10. Shi, H.-N.; Du, W.-S. Schur-convexity for a class of completely symmetric function dual. Adv. Theory Nonlinear Anal. Appl. 2019, 3, 74-89.

11. Shi, H.-N.; Du, W.-S. Schur-power convexity of a completely symmetric function dual. Symmetry 2019, 11, 897. [CrossRef]

12. He, L. Two new mappings associated with inequalities of Hadamard-type for convex function. J. Inequal. Pure Appl. Math. 2009, 10,81 . 